Relations industrielles

Industrial Relations

\title{
L'entreprise moderne et le management, par Pierre Schil, Paris, Entreprise Moderne d'Édition, 1969, 132 pp.
}

\section{Bernard Solasse}

Volume 25, numéro 1, 1970

URI : https://id.erudit.org/iderudit/028112ar

DOI : https://doi.org/10.7202/028112ar

Aller au sommaire du numéro

Éditeur(s)

Département des relations industrielles de l'Université Laval

ISSN

0034-379X (imprimé)

1703-8138 (numérique)

Découvrir la revue

Citer ce compte rendu

Solasse, B. (1970). Compte rendu de [L'entreprise moderne et le management, par Pierre Schil, Paris, Entreprise Moderne d'Édition, 1969, 132 pp.] Relations industrielles / Industrial Relations, 25(1), 155-155.

https://doi.org/10.7202/028112ar

Tous droits réservés @ Département des relations industrielles de l'Université Laval, 1970
Ce document est protégé par la loi sur le droit d'auteur. L’utilisation des services d'Érudit (y compris la reproduction) est assujettie à sa politique d'utilisation que vous pouvez consulter en ligne.

https://apropos.erudit.org/fr/usagers/politique-dutilisation/ 
The changes in the law have led to fewer, more restricted deferments. Undergraduate deferments were no longer discretionary. Deferments could no longer be compounded. Anyone deferred as a student, for example, was disqualified from deferment on grounds of fatherhood except in cases of extreme hardship. Graduate student deferments were restricted to those studying medicine or narrowly related subjects. As well, with these changes the problems of high induction age and longer draft uncertainty have been exacerbated.

The author concludes with suggestions to deal with the potential problem of the rising involuntary induction age. $\mathrm{He}$ also discusses alternative mechanisms for determining sequence of induction of 1-A registrants (by age group and by a lottery system) which implies relinquishing the oldest-first rule.

The selection procedures for the armed forces relate directly to both the efficiency of the armed forces and the use and allocation of manpower in the civilian economy. For this reason, this detailed paper merits the consideration of those involved in manpower plans.

\section{Joan SICHEL}

\section{L'entreprise moderne et le management,} par Pierre Schil, Paris, Entreprise moderne d'édition, 1969, 132pp.

Un livre qui se veut avant tout pratique et qui s'adresse aux chefs d'entreprises moyennes. L'optique est définie d'emblée dans l'avant propos: l'entreprise moyenne - 200 ouvriers ou moins s'adaptera ou disparaîtra. Cette évolution doit répondre à deux séries d'objectifs d'ailleurs complémentaires, soit "d'une part, un accroissement de productivité de rendement, en même temps qu'un accroissement de production (et) d'autre part, une promotion sociale qui attribue à chacun, à tous les échelons, une part importante des fruits du travail et de ses responsabilités" (p. 127). Il n'y a pas d'autre alternative possible et rationnelle.

Bien que conçu en fonction d'un contexte qui n'est pas le nôtre, en 1963 l'on comptait en France que 35 établissements comprenant plus de 5,000 salariés (p. 18). Cet ouvrage qui se veut simple et qui l'est, devrait intéresser plus d'un patron québécois par sa clarté et les réflexions pertinentes de l'auteur concernant les fonctions du chef d'une entreprise moyenne quelles soient d'ordre économique, organisationnel ou social.

Pour le scientifique et plus particulièrement pour le sociologue intéressé aux relations industrielles, la lecture de cet ouvrage est réconfortante à un autre titre puisqu'il constitue l'indice possible d'un renouvellement substantiel et prometteur du comportement et des préoccupations du chef d'entreprise moyenne que l'on souhaiterait largement partagé par ses pairs dans la mesure où il rompt avec le conformisme intransigeant d'un milieu social plus soucieux de survivre à n'importe quel prix que de travailler à l'expansion économique de l'ensemble et de maintenir un système de relations de travail périmé parce que marqué du sceau du paternalisme quand il ne tend pas à l'assujetissement des salariés.

\section{Bernard SOLASSE}

The Exceptional Executive, a Psychological Conception, by Harry Levinson, Cambridge, Mass., Harvard Business Press, second printing, 1969, 297 pp.

Cet ouvrage s'adresse d'abord aux administrateurs. L'auteur a voulu mettre à leur disposition une synthèse des contributions de la théorie, de la recherche et de l'expérience qui leur permette de mieux comprendre les exigences du leadership et les effets de son exercice sur les hommes, l'organisation et le milieu.

En appliquant la théorie psychanalytique aux notions de leadership, de travail et d'organisation, l'auteur leur donne une signification nouvelle, ou du moins originale, et qui a le mérite de permettre de mieux situer les contributions des autres théories psychologiques et administratives qui y vont chacune de leur point de vue sur ces sujets. Face à l'analyse de Levinson, un bon nombre de clichés, de slogans et d'idées toutes faites s'estompent. Pour l'auteur, il n'existe pas de solutions pré-déterminées aux problèmes humains de l'organisation et seule la compétence d'administrateurs connaissant bien les mécanismes humains et la nature de l'organisation, permettra de trouver des solutions qui ont bien des chances d'être différentes dans chaque cas. Levinson, 